\title{
Management von Innovationsprozessen für nachhaltige Entwicklung
}

\author{
Ein Wandel in Richtung Nachhaltigkeit erfordert \\ Innovationen in vielen Bereichen wirtschaftlichen \\ und gesellschaftlichen Handelns. Ein neues \\ Instrument erleichtert die Steuerung der notwen- \\ digen Innovationsprozesse. \\ Von Martina Schäfer, Bettina König, Anett \\ Kuntosch, Beate Richter und Tamara Schaal
}

\section{Einleitung}

Nachhaltigkeitsinnovationen sind dadurch gekennzeichnet, dass sie unterschiedliche Zielstellungen vereinbaren: neben ökonomischer Tragfähigkeit spielen ökologische und soziale Fragestellungen eine zentrale Rolle (Klewitz/Hansen 2014; Jänicke 2008). Die Herausforderung, sich gegenüber bestehenden Produkten, Dienstleistungen und Lösungsansätzen zu etablieren, ist bei Nachhaltigkeitsinnovationen besonders groß, da die vorherrschenden Rahmenbedingungen den ökologischen und sozialen Mehrwert bisher ungenügend honorieren (Clausen/Fichter 2019; Belz/Schrader 2012).

Beschäftigte in Unternehmen, Beratungsbüros, der Verwaltung oder Verbänden sowie Projekt-, Netzwerk-, Regional- oder Clustermanager sind häufig mit der Aufgabe konfrontiert, derartige Innovationsprozesse in einer Region, für eine innovative Nischenlösung oder für einen Sektor anzustoßen und zu koordinieren. Obwohl diesen koordinierenden Akteuren eine Schlüsselrolle in nachhaltigen Transformationsprozessen zugeschrieben wird, sind ihre Rollen doch sehr unterschiedlich und ihre Ressourcen oft nicht ausreichend, um alle notwendigen Rollen und Managementaufgaben abzudecken (Kivimaa et al. 2018; Ingram 2015; Richter 2019). Grundannahme unseres Beitrages ist, dass in der Gestaltung von Innovationsprozessen ein wesentlicher Ansatzpunkt liegt, um Nachhaltigkeitsinnovationen über die Nische heraus zu entwickeln und $\mathrm{zu}$ verbreiten. Dies beinhaltet, auf verschiedene Komplexitätsherausforderungen reagieren zu können (Garud et al. 2013).

In diesem Artikel wird zunächst auf die Herausforderungen des Managements von Nachhaltigkeitsinnovationsprozessen allgemein und aus einzelnen thematischen Perspektiven, die als erfolgskritisch diskutiert werden, eingegangen. So wiesen van de Ven und Engleman (2004) bereits 1986 auf vier Herausforderungen hin: die Aufmerksamkeit managen (Akzep- tanz und Marketing), die Prozessgestaltung von der Idee zum Umsetzungserfolg (Prozessgestaltung und Erfolgsabschätzung), das Verhältnis des Einzelnen und des Gesamten (Wissensmanagement) sowie institutionelle Führungsherausforderungen (Kooperationsmanagement).

Aufbauend auf den geschilderten Herausforderungen, wird eine Toolbox beschrieben, die entwickelt wurde, um diesen in der täglichen Managementpraxis zu begegnen. Der sogenannte ginkoo-Innovationsnavigator ist im Rahmen des ginkooProjektes entstanden [1]. Damit soll es den vorher genannten Akteuren ermöglicht werden, auf Probleme der Innovationsmanagementpraxis reagieren zu können. Der ginkoo-Innovationsnavigator wurde im Austausch mit Unternehmen und Organisationen des nachhaltigen Landmanagements beziehungsweise der Land- und Ernährungswirtschaft entwickelt. Er bietet aber auch Akteuren aus anderen Sektoren durch die enthaltenen Reflexions- und Planungswerkzeuge Anregungen dafür, wie sie Ansatzpunkte für die Gestaltung von Innovationsprozessen identifizieren können.

Ziel des ginkoo-Innovationsnavigators ist es, die Nutzer/innen im Innovationsprozess zu unterstützen, Herausforderungen hinsichtlich der Berücksichtigung von Nachhaltigkeitsaspekten, des Einbezugs von Kundenbedürfnissen sowie des Kooperations- und Wissensmanagements zu identifizieren und mithilfe von Tools situationsangepasste Managementstrategien zu entwickeln. Die Tools unterstützen bei Fragen, wie es gelingt, effizient miteinander zu kooperieren, sich über nachhaltige Lösungen zu verständigen, wie die Akzeptanz für die Innovation erhöht oder wie Wissen über unterschiedliche Gruppen von Akteuren hinweg genutzt, die Erfolgschancen eingeschätzt und Marketing- und Kommunikationsmaßnahmen dafür konzipiert werden können.

\section{Innovationsmanagement als komplexe Herausforderung}

In der Innovationsforschung wird seit Längerem davon ausgegangen, dass Innovationsprozesse keinem linearen Modell von Generierung, Weiterentwicklung und Verbreitung folgen, sondern in rekursiven Schleifen verlaufen (Garud et al. 2013; Schroeder et al. 2000). Die Innovationsforschung reagiert damit auf die empirische Beobachtung, dass neue Ideen interpretativ flexibel sind und im Laufe ihrer Weiterentwicklung oft verschlungene, sowohl divergierende als auch konvergierende Pfade einschlagen (Lettkemann 2018, S. 2). Prominente Modelle, um die nichtlineare Dynamik und Kontingenz solcher In- 
novationsprozesse zu erklären, sind beispielsweise das Feuerwerksmodell (Schroeder et al. 2000) und die innovation journey (Van de Ven et al. 1999), die wiederkehrende Muster bei der Initialisierung, Entwicklung und Implementation von Innovationen beschreiben. Dabei wird davon ausgegangen, dass Ideen nicht in einem einmaligen Akt der Invention entstehen, sondern sie sich auch während der Entwicklung und Implementierung wandeln und ausdifferenzieren (Van de Ven 2008). Auf die Dynamik von Innovationsprozessen und ihrem Verlauf in iterativen Schleifen mit wiederkehrenden Phasen wird von verschiedenen Autor/innen verwiesen. In diesen Phasen begegnen den Akteuren Hindernisse, es wird auf veränderte Umfeldbedingungen und Akteursinteressen reagiert und es werden jeweils angepasste Lösungen erprobt (Knierim et al. 2015; Ingram 2015; Richter et al. 2020).

Die Literatur verdeutlicht, dass Innovationen in Gegenwartsgesellschaften nicht von einzelnen Unternehmerpersönlichkeiten geschaffen werden, sondern Produkte organisierter Zusammenarbeit darstellen (Garud et al. 2013; Ndah et al. 2017). Sie werden als in Netzwerken von Akteuren stattfindende soziale, institutionelle sowie technische Prozesse beschrieben, in denen interaktives Lernen entlang eines gemeinsamen Themas oder Veränderungsimpulses stattfindet (Knierim et al. 2015; Hurmelinna-Laukkanen/Nätti 2018). Hieraus ergibt sich die Notwendigkeit für Kooperationen, denn angesichts hoher Unsicherheit bezüglich technologischer, organisationaler und marktlicher Erfolgsaussichten mit der Etablierung von Innovationen ist ein erheblicher Ressourcenaufwand verbunden, den einzelne Akteure alleine nicht aufbringen können (Van de Ven et al. 2008). Durch begrenzte Ressourcen von kleinen und mittelgroßen Organisationen und Unternehmen werden für die Umsetzung von Innovationsvorhaben außerdem oftmals Expertise und Wissen aus unterschiedlichen Quellen benötigt, um es interorganisational weiterzuentwickeln und nutzen zu können.

Aufgrund dieser Herausforderungen in Innovationsprozessen führen rezeptartige Empfehlungen oder Tools für das Managen nicht unbedingt zum Innovationserfolg (Zillner/Krusche 2012), da Komplexität nicht kontrolliert werden kann, sondern als Herausforderung angenommen werden muss (Garud et al. 2013). Dabei spielen das Ermöglichen des Lernens aus Fehlern durch Monitoring und Evaluation (Wielinga et al. 2010) sowie ein konstruktiver Umgang mit temporären Organisationsformen (Sydow/Braun 2018) eine Rolle, um Spielraum für neue Entwicklungen zu gewährleisten.

Bei der Entwicklung des ginkoo-Innovationsnavigators wurde diesen Aspekten Rechnung getragen, indem ermöglicht wird, verschiedene Perspektiven gleichzeitig im Blick zu behalten. Konzipiert wurde der ginkoo-Innovationsnavigator als eine Zusammenstellung von Instrumenten, die vorrangig die periodische Selbstreflektion des Innovationsmanagements beziehungsweise der Gruppe der Akteure, die für die erfolgreiche Etablierung einer Nachhaltigkeitsinnovation kooperieren, unterstützt.

\section{Zentrale thematische Perspektiven für das Innovationsmanagement}

In der Literatur werden verschiedene Perspektiven oder thematische Schwerpunktthemen als erfolgsrelevant für Nachhaltigkeitsinnovationsprozesse benannt, auf die im Folgenden als Grundlage für den entwickelten Innovationsnavigator eingegangen wird.

Ziele haben eine wichtige Funktion. Sie geben Orientierung, reduzieren Unsicherheit, motivieren die Beteiligten, ermöglichen Entscheidungen, Koordination und Kontrolle (Tidd/Bessant 2013). Nachhaltigkeitsinnovationen zeichnen sich einerseits durch ihre normative Richtung, etwa Nachhaltigkeitsziele, und die jeweils neu zu entwickelnde Lösung, wie Innovationsziele, im jeweiligen Kontext aus. Damit Nachhaltigkeit verbessert werden kann, genügt es oft nicht, Bestehendes durch inkrementelle Innovationen wie Effizienzsteigerung besser zu machen. Häufig müssen Dinge anders getan werden. Notwendig sind radikale Innovationen, wie zum Beispiel Suffizienzlösungen. Wie dies geschehen kann, erfordert die Formulierung und Operationalisierung von Nachhaltigkeits-, Innovationsund Umsetzungszielen (Sydow/Braun 2018). Da es während des Innovationsprozesses immer wieder zu fragilen Phasen kommt, ist eine regelmäßige Prüfung der Zielsetzungen in Abstimmung zwischen den beteiligten Akteuren elementar.

Nachhaltigkeitsinnovationsprozesse erfordern die Beteiligung unterschiedlicher Akteure mit abweichenden Normen und Werten. Da diese Werte nicht transparent für alle Beteiligten sind, können unterschiedliche Wahrnehmungen über die Art des Problems bestehen und - damit verbunden - divergierende Zielsetzungen und Ideen, wie Nachhaltigkeit erreicht werden kann (Blok et al. 2016; Geels/Schot 2007). Blok et al. (2016) legen dar, dass für die Behandlung von Nachhaltigkeitsthemen sowohl normative als auch Handlungskompetenzen notwendig sind. Normative Kompetenzen umfassen, dass die Beteiligten in der Lage sind, Nachhaltigkeitsthemen zu erkennen, und abschätzen können, wie sie mit ihren individuellen ethischen Normen im Austausch mit anderen Akteuren umgehen. Dabei geht es darum, die Verantwortung dafür zu akzeptieren, dieses Thema gemeinsam zu adressieren. Da es sich bei der Festlegung von Nachhaltigkeitszielen und -maßnahmen letztlich um Aushandlungsprozesse handelt, erscheint es sinnvoll, dass lokale oder regionale Akteure in ihre Formulierung einbezogen werden und sich darüber austauschen können (Reed et al. 2006; Koutsouris 2008; Hermans et al. 2010; Orenstein et al. 2017). Für die Koordination von Innovationsprozessen sind Entscheidungsunterstützungssysteme hilfreich, die die Übereinstimmung von Zielen zwischen den Akteuren und deren Kommunikation bis hin zu den Konsument/innen befördern (Zoll et al. 2019).

Kunden-, aber auch Nutzerorientierung von Innovationen ist ein zentraler Erfolgsfaktor in Innovationsprozessen (Rogers 2013). Akzeptanz wird im Zusammenhang mit Innovationsprozessen einerseits im Hinblick auf die beteiligten Mit- 
arbeiter/innen in Unternehmen und andererseits hinsichtlich der Zielgruppe, wie etwa der Kund/innen von Produkten und Dienstleistungen, Anwender/innen, aber auch der Betroffenen, zum Beispiel von neuen Technologien, thematisiert.

Die Unsicherheit hinsichtlich der Kundenakzeptanz ist einer der zentralen Hemmnisfaktoren für den Beginn von Innovationsprojekten (Rammer et al. 2006). Um diesem Risiko zu begegnen, werden vermehrt Formen der Integration „fortschrittlicher" Kund/innen und Nutzer/innen in frühe Phasen des Innovationsprozesses zur Sicherung der späteren Kundenakzeptanz, zur Reduzierung der Floprate von Innovationsvorhaben und zur erfolgreichen Diffusion von Innovationen diskutiert (Fichter 2005; Busse/Siebert 2017). In der Debatte um die Einführung von neuen Technologien, wie zum Beispiel Formen erneuerbarer Energien, wird dagegen stärker die Notwendigkeit partizipativer Maßnahmen etwa auf lokaler oder regionaler Ebene betont, um die Akzeptanz derjenigen zu erhöhen, die durch negative Auswirkungen beeinträchtigt werden, ohne unmittelbar von der Technologie zu profitieren. Die Öffnung von Innovationsprozessen für verschiedene Anspruchsgruppen ist aus Managementsicht sehr voraussetzungsvoll (Tidd/Bessant 2013). Im ginkoo-Innovationsnavigator wurde der Bedarf für Methoden der Akzeptanzanalyse und Instrumente der Akzeptanzsteigerung berücksichtigt, die auch bei geringen Zeitressourcen eingesetzt werden können (Busse et al. 2019a).

In Innovationsprozessen ergibt sich die Notwendigkeit zur Kooperation daraus, dass viele technische und organisationale Probleme, die mit der Entwicklung und Implementation von neuen Ideen einhergehen, zu komplex sind, um sie isoliert und von einzelnen Akteuren mit begrenzter Ressourcenausstattung bearbeiten zu können (Van de Ven et al. 2008). Als Vorteile von Innovationsnetzwerken werden der gegenseitige Knowhow-Transfer, die reziproke Bereitstellung von Ressourcen sowie das Erschließen neuer Marktsegmente mit neuen Partnern benannt (Sydow 1992, Hirsch-Kreiensen 2002). Durch das Erzielen von Synergieeffekten können Entwicklungsprozesse beschleunigt werden (Fichter et al. 2006). Für Nachhaltigkeitsinnovationen spielen Kooperationen eine besonders wichtige Rolle, da es häufig um Systemlösungen geht, für die auch neue institutionelle Arrangements notwendig sind. Um die besondere Qualität von nachhaltigeren Produkten und Dienstleistungen gewährleisten und transparent darstellen zu können, sind Abstimmungen und Qualitätssicherung über die Wertschöpfungskette oder über das Innovationsnetzwerk hinweg erforderlich. Als Voraussetzungen beziehungsweise Erfolgsfaktoren werden gemeinsame Zielvorstellungen, kompatible Unternehmenskulturen sowie gegenseitiges Vertrauen benannt (ebd.).

In der Literatur wird zwischen vertikalen Kooperationen entlang der Wertschöpfungskette oder horizontaler Zusammenarbeit z. B. zwischen Akteuren aus der Wirtschaft, Zivilgesellschaft und Verwaltung differenziert (Sydow 2006). Es werden außerdem verschiedene Phasen der Netzwerkbildung unterschieden (Anbahnungs- und Aufbauphase, Entwicklungs- phase, Realisations- und Wandlungsphase), in denen sich unterschiedliche Anforderungen an das Netzwerkmanagement stellen (Koller et al. 2006). Diese grundlegenden Erkenntnisse wurden für die Entwicklung von Tools im Rahmen des ginkooInnovationsnavigators und des Kooperationsleitfadens genutzt (Nölting/Schäfer 2020; Schäfer et al. 2019).

Relevantes Wissen über verschiedene Organisationseinheiten und aus unterschiedlichen Wissensbeständen im Innovationsprozess zu organisieren, gehört zu einer der zentralen Perspektiven des sogenannten, Orchestrierens ' von Innovationen (Dhanaraj/Parkhe 2006; Hurmelinna-Laukkannen 2018). Die praktische Notwendigkeit dafür ergibt sich aus dem Potenzial des Öffnens des Innovationsprozesses, um durch die Integration verschiedener Wissensbestände Innovationsrisiken zu minimieren (Chesbrough 2006; Peterson/Mager 2011). In der Praxis für Nachhaltigkeitsinnovationen stellt es eine Herausforderung dar, wenn die dafür notwendige Funktion von keinem der beteiligten Akteure übernommen wird (Kuntosch et al. 2020). Allgemein wird Wissensmanagement häufig enthusiastisch begonnen, die Ergebnisse bleiben aber oftmals hinter den Erwartungen zurück und der konkrete Erfolg einzelner Maßnahmen kann nur schwer bewiesen werden (McIver/Lengnick-Hall 2018). Das liegt üblicherweise daran, dass Wissensmanagement unter schwierigen Rahmenbedingungen durchgeführt wird, wie zum Beispiel unzureichende Managementunterstützung, Planung, Koordination und Evaluierung der gewählten Maßnahmen, unzureichende Qualifikation der verantwortlichen Mitarbeiter/innen und eine schlechte Ressourcenausstattung (Probst et al. 2012).

Mit der Einsicht, dass Innovationsprozesse über gesellschaftliche Bereiche und Organisationen hinweg organisiert werden müssen (Leydesdorff/Etzkowitz 1996), geht auch die Notwendigkeit einer angepassten Vorgehensweise für das Marketing einher (Pronay/Buzas 2015). In Innovationsprozessen wird dem Marketing oftmals nur eine geringe Bedeutung beigemessen oder es wird erst am Ende aufgegriffen. Da sich der Erfolg von Marketingmaßnahmen erst zeitlich versetzt einstellt, werden häufig ungenügend finanzielle und personelle Ressourcen für das Marketing in Innovationsprozesses eingeplant (Hofbauer et al., 2009). Nachhaltigkeitsinnovationen können ohne Marktkenntnisse und zielgerichtetes Marketing nicht bestehen, wenn sie sich gegenüber bestehenden Lösungen durchsetzen wollen (Trommsdorf/Steinhoff, 2013; Trommsdorf/Binsack 1999). Auch gute und durchaus sinnvolle und notwendige Innovationen verschwinden teilweise nach kurzer Einführung wieder vom Markt, da die Nachfrage und/oder der Absatz zu gering sind (Albers 2005; von Ahsen et al. 2010). Dabei werden Verbraucher/innen oftmals aufgrund fehlender oder unzureichender Kommunikations- und Marketingmaßnahmen auf die Innovationen schlicht nicht aufmerksam (Weiber/Pohl 2017).

Innovationsmarketing, das den Marketingprozess mit dem Innovationsprozess verschränkt, kann wesentlich zum Erfolg einer Innovation beitragen. Wie Marketing- und Innovations- 
management praktisch aufeinander abgestimmt werden können, ist in komplexen Innovationsprozessen eine Managementherausforderung (Steinhoff/Trommsdorff 2011).

Es gibt verschiedene Bewertungsansätze, um mit der Unsicherheit hinsichtlich des Erfolgs oder Misserfolgs, der Innovationen innewohnt, informiert umgehen zu können. Diese müssen einerseits berücksichtigen, um welche Innovationsform es sich bei der zu betrachtenden Nachhaltigkeitsinnovation handelt. So sprechen Tidd und Bessant (2013) beispielsweise von Produkt-, Dienstleistungs-, Positions- und Paradigmainnovationen und Corsten et al. (2016) von Produkt-, Verfahrens- und Sozialinnovationen. Neben der Verschiedenartigkeit von Innovationen und im Hinblick auf ihren Beitrag für eine nachhaltige Entwicklung (Möller et al. 2011) sind betriebswirtschaftliche Bewertungskriterien wichtig, damit die anderen beabsichtigten Nachhaltigkeitswirkungen erreicht werden können. Durch Umdeutungen, Übersetzungen, Rekonfiguration von Innovationen im Verlauf von Innovationsprozessen verändert sich die Beschreibung und Bewertung des Entwicklungsfortschritts (Garud et al. 2013). Akteure stehen daher im Licht von situativer Komplexität immer wieder vor der voraussetzungsvollen Aufgabe abzuschätzen, ob weitere Innovationsarbeiten wirtschaftlich vertretbar geleistet werden können.

\section{Das Innovationsmanagement-Tool: der ginkoo-Innovationsnavigator}

Mit dem ginkoo-Innovationsnavigator können Innovationsprozesse reflektiert und geplant werden [2]. Dabei kann der ginkoo-Innovationsnavigator sondierend alleine durch den/die jeweilige/n Innovationsmanager/in genutzt werden. Bei vielen Fragen bietet sich aber eine gemeinsame Klärung in der Gruppe der Akteure an, die für die Entwicklung des Innovationsprozesses verantwortlich ist.

Mit dem ginkoo-Innovationsnavigator können Innovationsvorhaben aus mehreren Perspektiven schrittweise unterschiedlich intensiv bearbeitet werden.

Dazu zählen:

I sich zu den verschiedenen Perspektiven Zielformulierung, Nachhaltigkeitsbewertung, Akzeptanz, Kooperation, Wissensmanagement, Marketing und Innovationsbewertung informieren,

I Lösungen mit dem Basischeck aus verschiedenen Perspektiven überblicksartig reflektieren,

I mithilfe von Tools gezielt Knackpunkte bearbeiten,

I Ansatzpunkte klären,

I die nächsten Schritte planen und

I konkrete Maßnahmen umsetzen.

\subsection{Nutzungsmöglichkeiten}

$\mathrm{Zu}$ Beginn werden der Nutzen des ginkoo-Innovationsnavigators, die Funktionsweise und der Basischeck beschrieben sowie die im Navigator enthaltenen Themen und Tools. Im „Kompass“ wird jeweils das Thema kurz erläutert, was das Tool leisten kann

\section{„Einfache Empfehlungen oder \\ Instrumente für das Steuern \\ von Innovationsprozessen führen \\ nicht unbedingt zum Erfolg, \\ da Komplexität \\ nicht kontrolliert werden kann. “}

und es werden Beispiele aus dem ginkoo-Projekt vorgestellt. Zitate aus der Testphase illustrieren Anwendungsmöglichkeiten und -nutzen. Zu einzelnen Themenbereichen besteht die Möglichkeit, sich sehr umfangreich und vertieft zu informieren.

Mit dem Basischeck kann ein Überblick zum Innovationsprozess erstellt werden, indem aus verschiedenen Perspektiven der Stand des Vorgehens beschrieben und bewertet wird. Der Basischeck wird als Minimal-Variante empfohlen.

I Was wird in den Bereichen bereits umgesetzt?

I Und wie wirkt sich das bisherige „Managen“ jeweils auf das Innovationsgeschehen aus?

- Gibt es ein Problem und in welchem Bereich stört es das Innovationsgeschehen besonders?

Der Basischeck dokumentiert die aktuelle Situationseinschätzung und gibt allgemeine und detaillierte Hinweise zu Handlungsbedarfen. Aus diesem Überblick kann der Plan für das Navigieren im nächsten halben oder ganzen Jahr auf einem Arbeitsblatt überblicksartig erstellt werden. Nach einigen Monaten oder nach einer Toolanwendung kann eine neue Version erstellt und gespeichert werden. Im Team oder mit den weiteren Beteiligten genutzt, haben alle schnell den gleichen Wissensstand als Basis für die gemeinsame Weiterentwicklung. Da der Basischeck eine Selbsteinschätzung ist, lohnt es sich, die Ergebnisse im Austausch mit wichtigen Beteiligten oder auch kritischen Akteuren zu überprüfen, um blinde Flecken zu vermeiden.

Aus der Gesamt- und Einzelauswertung des Basischecks erfahren die Nutzer/innen, in welchem der sieben Bereiche die Situation als besonders riskant eingeschätzt wurde. Die Einzelauswertung der Fragen gibt Hinweise darauf, wo Handlungsbedarf besteht und welche Einzeltools sich eignen, um diese Knackpunkte zu bearbeiten.

Nach der Anwendung eines Einzeltools wurde idealerweise neues Wissen erarbeitet, zum Beispiel gemeinsame „AHA-Effekte “, die zu einem neuen Verständnis der Situation beitragen und das Planen und Organisieren der nächsten Schritte im Innovationsgeschehen vereinfachen. Die Einzeltools bieten zum Teil Dokumentationshilfen an, um die Ergebnisse festzuhalten. 


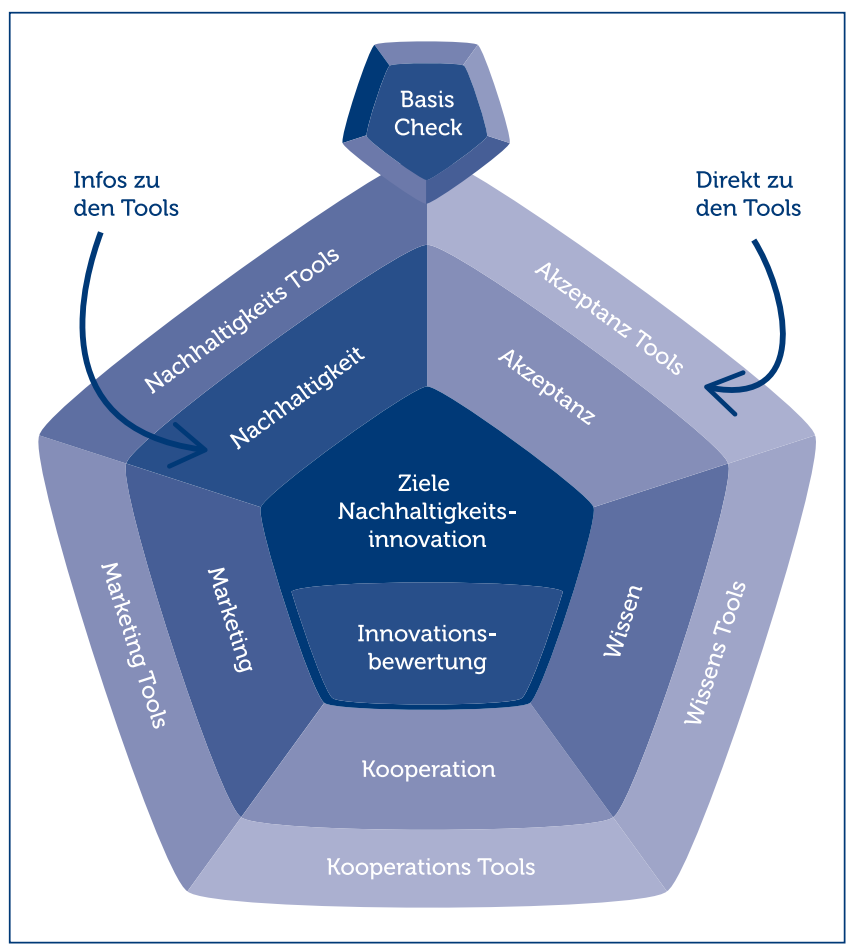

Abbildung 1: 2D-Perspektive auf den ginkoo-Innovationsnavigator [2]

(Grafik: Harald Junker)

Mit dem erweiterten Wissen zu einem Einzelbereich können Umsetzungsschritte entwickelt werden, wie Marketingmaßnahmen, Maßnahmen zur Verbesserung der Akzeptanz oder der Kooperation der beteiligten Akteure. Diese Umsetzungsschritte sollten vor der konkreten Planung jedoch immer wieder mit den Nachhaltigkeits-, Innovations- und vor allem den Umsetzungszielen abgeglichen werden.

Als Hilfestellung für die gemeinsame Planung in heterogenen Akteurskonstellationen bietet der ginkoo-Innovationsnavigator ein an den Themenkompass angelehntes Arbeitsblatt an. Es kann direkt nach dem Basischeck oder nach der Bearbeitung einzelner Knackpunkte genutzt werden, um die nächsten Schritte festzuhalten. Komplexere Vorhaben können auch als eine Art Rundumpaket mit mehreren aufeinanderfolgenden Toolanwendungen konzipiert werden. Wichtig ist, dass jemand die Verantwortung für die Umsetzung dieses Plans übernimmt und die Ergebnisse zu einem vereinbarten Zeitpunkt überprüft werden. Je umfangreicher der Plan wird, umso wichtiger ist es zu prüfen, ob die Umsetzung allein gelingen kann oder Feedback und Unterstützung benötigt werden.

Im letzten Schritt können nun in dem Bereich Maßnahmen gezielt und koordiniert umgesetzt werden. Herausforderung in der Gestaltung von Innovationsprozessen ist, dass die Zeithorizonte für bestimmte Arbeiten unterschiedlich lang sein können und verschiedene Maßnahmen parallel von unterschiedlichen Akteuren umgesetzt werden müssen. Die einzelnen Maßnahmen müssen daher von der Koordination immer wieder in den Gesamtzusammenhang gebracht und kommuniziert wer- den. Diese Herausforderung erhöht sich, wenn mehrere Innovationsansätze gleichzeitig verfolgt werden, die jeweils verschiedene Umsetzungsziele haben.

\subsection{Themen und Tools im ginkoo-Innovationsnavigator}

Wie oben bereits ausgeführt, deckt der ginkoo-Innovationsnavigator verschiedene Perspektiven ab, die bei der Entwicklung von komplexen Nachhaltigkeitsinnovationen erfolgskritisch sind. Als fünfeckiger Kompass aufgebaut, ermöglicht die Struktur, eine Lösung aus verschiedenen Perspektiven zu betrachten und an den aktuell kritischen Knackpunkten zu bearbeiten (s. Abb. 2).

Im Rahmen des ginkoo-Innovationsnavigators werden die Teilnehmer/innen dazu motiviert, die unterschiedlichen Zielstellungen explizit $\mathrm{zu}$ machen und periodisch $\mathrm{zu}$ überprüfen. Nachhaltigkeitsziele lenken das Innovationsgeschehen. Für die Ausrichtung und Planung zielführender Maßnahmen sind sie unerlässlich. Innovationsziele sind Ansatzpunkte und Lösungsideen, mit denen die Nachhaltigkeitssituation durch eine Kombination aus Neuem, Veränderung und Bewährtem vor Ort verbessert werden soll. Es kann sich um Technologien, Organisationsformen, soziale Praktiken sowie Kombinationen davon handeln. Umsetzungsziele sind eine notwendige Voraussetzung, damit die beteiligten Akteure sich über Unternehmens-, Verwaltungs- und andere Grenzen hinweg organisieren können. Für ein Innovationsziel können mehrere Umsetzungsziele wichtig sein und zu jedem dieser Umsetzungsziele müssen manchmal unterschiedliche Akteure kooperieren, Wissen zusammentragen und kommunizieren. Unkonkrete oder unvollständige Nachhaltigkeits-, Innovations- und Umsetzungsziele führen zu Situationen von Unsicherheit, Unübersichtlichkeit und Unzufriedenheit über das Fortbestehen von Nachhaltigkeitsproblemen.

Die Ziele sollten immer wieder überprüft werden. Insbesondere die Operationalisierung von Umsetzungszielen ist eine wichtige Aufgabe. Idealerweise mündet die Anwendung eines Tools aus den anderen Perspektiven, zum Beispiel die Erstellung einer Wissenslandkarte, in konkrete Umsetzungsziele für die nächsten Schritte. Unter allen beteiligten Akteur/innen abgestimmte Nachhaltigkeitsziele schaffen eine gemeinsame Vision. Sie machen den Mehrwert der Innovation sichtbar und ermöglichen eine Zielkontrolle.

Da die Definitionen von Nachhaltigkeit der an der Innovation beteiligten Akteure nicht immer übereinstimmen, motiviert das im Rahmen des ginkoo-Innovationsnavigators bereitgestellte Tool zur Klärung, wer welche Nachhaltigkeitsziele verfolgt, welche die wichtigsten Ziele sind und inwiefern sie umgesetzt werden. Dadurch kann konkretisiert werden, was die Innovation zu ökologischer, ökonomischer und sozialer Nachhaltigkeit beiträgt.

Mit dem Onlinetool können gemeinsam mit allen beteiligten Akteuren die Nachhaltigkeitskriterien ausgewählt werden, die am wichtigsten für den Mehrwert der Innovation sind, und es kann gewichtet werden, inwieweit die Kriterien bereits 
erfüllt werden. Beides wird in Form einer Spinnengrafik dargestellt, sodass die Einschätzungen unterschiedlicher Teilnehmer/innen oder Akteursgruppen miteinander verglichen werden können (Zoll et al. 2020). Die Ergebnisse können im weiteren Verlauf dazu genutzt werden, unterschiedliche Gewichtungen in der Gruppe der Beteiligten zu diskutieren. Sie können auch als Ausgangspunkt dafür dienen, die Kommunikation nach außen so zu gestalten, dass potenzielle Kund/innen oder Nutzer/innen den sozialen, ökologischen und ökonomischen Mehrwert auch wahrnehmen.

Wie oben dargestellt, ist die Akzeptanz vonseiten der beteiligten Akteure ein wichtiger Aspekt, der zum Erfolg von Innovationen und Projekten beiträgt. Werden Innovationen von Nutzer/innen nicht angenommen, können sie keinen langfristigen Erfolg haben. Ablehnung kann zusätzlich zu erheblichen Konflikten führen, zum Beispiel, wenn das Projekt auf aktive Gegnerschaft trifft (Busse et al. 2019 a, b). Ohne das Wissen, welche Umstände und Aspekte die Akzeptanz bestimmen, kann diese allerdings nicht verbessert und können Probleme nicht abgemildert oder behoben werden. Die entwickelten Tools helfen daher dabei, Akzeptanzprobleme zu erkennen und geben Anregungen für akzeptanzsteigernde Maßnahmen (Busse et al. 2019c). Im Idealfall werden sie schon zu Beginn der Innovationsentwicklung genutzt, um auf potenzielle Probleme und fördernde Aspekte von Beginn an eingehen zu können. Aber auch im weiteren Verlauf ist eine Anwendung sinnvoll, um auf auftretende Probleme angemessen reagieren zu können. Das Tool bietet ein Set an Bausteinen wie fact sheets zu Erhebungsmethoden und Partizipationsmethoden zur Akzeptanzsteigerung, die für verschiedene Bedarfe genutzt werden können.

Wie oben dargestellt, ist Zusammenarbeit mit anderen Akteuren in der Regel notwendig, um Innovationen für nachhaltiges Landmanagement erfolgreich zu etablieren. Eine der zentralen Fragen ist, welche Mischung an Akteuren sinnvoll und notwendig ist, um die Innovation mit den angestrebten Nachhaltigkeitsleistungen langfristig etablieren zu können. Für ein erfolgreiches Miteinander sind die gemeinsamen Ziele, die Arbeitsteilung sowie eine faire Verteilung von Aufwand und Ertrag zu klären. Kooperationen benötigen außerdem ein Minimum an Managementstrukturen, um eine stabile Zusammenarbeit zu gewährleisten, die auf Vertrauen basiert. Das Management dieser Kooperationen ist durch teilweise widersprüchliche Ziele - ökonomische Tragfähigkeit bei gleichzeitiger Erbringung von Nachhaltigkeitsleistungen - sehr anspruchsvoll. Die im ginkoo-Innovationsnavigator und im Leitfaden Kooperationsmanagement (Schäfer et al. 2019) zur Verfügung gestellten Tools unterstützen die Akteure, die Kooperationen initiieren oder organisieren, dabei, ihre Zusammenarbeit erfolgreich zu gestalten. Die Unterstützungsangebote sind mehrstufig aufgebaut. Neben einer schnellen Orientierung, an welchen Punkten Unklarheit oder Handlungsbedarf bestehen, bietet der Leitfaden mehrere Einzelinstrumente, die es den Koordinator/innen ermöglichen, offene Fragen für sich oder gemeinsam mit anderen zu klären.

\section{„Relevantes Wissen über verschiedene Organisationseinheiten und aus unterschiedlichen Wissensbeständen im Innovationsprozess zu organisieren, gehört zu einer der zentralen Herausforderungen in der Steuerung von Innovationen."}

Wie oben dargestellt, kann Wissensmanagement durch gute Planung und Abstimmung mit anderen Managementaufgaben entscheidend zum Erfolg des Innovationsvorhabens beitragen. Die Roadmap Wissensmanagement bietet mit Tools und Tipps Unterstützung dabei, diese Ressource für Innovationsprozesse effektiver zu nutzen (Kuntosch 2019). Dazu gehört, einen Überblick über die wichtigen Wissensbestände, -lücken und -monopole bei einzelnen Akteuren zu gewinnen und Wissensziele regelmäßig auf ihre Aktualität hin zu überprüfen. Dabei besteht eine besondere Herausforderung darin, den für das Innovationsvorhaben notwendigen Wissensprozess an den Schnittstellen zwischen Funktionen und Akteuren gut aufeinander abzustimmen und dafür notwendige Rahmenbedingungen und Herangehensweisen zu schaffen. Bei den Tools aus den Bereichen Strategisches und Operatives Wissensmanagement handelt es sich vor allem um analoge, kommunikationsfördernde Instrumente, da es sich gezeigt hat, dass Software die Komplexität von Nachhaltigkeitsproblemen und komplexen Prozessen (noch) nicht hinreichend abbilden kann. Der angebotene Mix aus Tool-Anleitungen und Kurz-Checklisten, die logisch aufeinander aufbauen, soll eine effektive Gestaltung von Wissensprozessen ermöglichen.

Mit passgenauem Marketing kann auf Innovationen aufmerksam gemacht und die Innovationsidee gestärkt werden. Marketing umfasst zugleich unternehmerisches Planen und Handeln und orientiert sich dabei am Markt und den Nutzer/innen. Aufgrund der Schnelllebigkeit des Marketings und der Individualität von Unternehmen beziehungsweise weiteren Organisationen können im Rahmen des Tools keine maßgeschneiderten Marketingstrategien vorgeschlagen, sondern nur der Weg dahin aufgezeigt werden. Marketingstrategien sollten jeweils individuell auf die jeweilige Innovation und Zielgruppe zugeschnitten sein. Das Tool leistet Hilfestellung im Hinblick auf die Integration von Marketing in Innovationsprozesse und dem allgemeinen Verständnis für Marketing (Richter/König 2019). Es werden Grundlagen zum Marketing sowie die strategische Umsetzung und die Einplanung finanzieller sowie personeller Ressourcen vermittelt. 
Das Invention Assessment Tool (IAT) erlaubt bereits in der Anfangsphase des Innovationsprozesses eine Prognose hinsichtlich der Erfolgsaussichten der Innovation. Das IAT kann anhand von wirtschaftlichen, sozialen und ökologischen Indikatoren Schwachstellen und Hemmnisse aufzeigen, die eine erfolgreiche Implementierung wesentlich beeinflussen. Es bildet einen idealtypischen Innovationsprozess anhand der verschiedenen Innovationstypen (Produkt-, Prozess-, Marketingund Organisationsinnovation) ab.

Mithilfe des Bewertungsergebnisses wird ein Überblick gegeben, in welchen erfolgsrelevanten Bereichen Verbesserungspotenzial für die geplante Innovation besteht. Dabei ist es sinnvoll, das IAT bereits zu Beginn des Innovationsprozesses anzuwenden, um früh Hinweise auf Verbesserungsbedarfe zu erhalten. Der individuelle Bewertungsprozess und das Bewertungsergebnis werden gespeichert und sind jederzeit abrufbar. Das IAT kann im Innovationsprozess fortlaufend als Kontrollinstrument genutzt werden, in dem der Bewertungsprozess wiederholt und Veränderungen im Ergebnis im Vergleich zur Ausgangssituation reflektiert und ggf. Anpassungen vorgenommen werden. Dabei werden auch Schwachstellen erkennbar, auf die mit den Tools aus den anderen Perspektiven reagiert werden kann.

\section{Erprobung des Tools und Ausblick}

Der ginkoo-Innovationsnavigator als Ganzes und die Tools in den einzelnen Bereichen wurden im Rahmen des transdisziplinären Projekts ginkoo im engen Austausch mit den beteiligen Praxisakteuren aus der Land- und Ernährungswirtschaft und dem nachhaltigen Landmanagement entwickelt. Einige der Einzeltools wurden in den zwei betrachteten Fallstudien erprobt und auf Basis der gewonnenen Erfahrungen optimiert. Insbesondere wurde aber der Aufbau des ginkoo-Innovationsnavigators in mehreren Stufen sowie die Handhabbarkeit und Verständlichkeit mit den Praxisakteuren in iterativen Entwicklungsschleifen rückgekoppelt. In einem weiteren Schritt wurden Tooltests mit unterschiedlichen Akteuren aus dem nachhaltigen Landmanagement vorgenommen und das Feedback in Überarbeitungsschritte einbezogen.

Übereinstimmend wurde von den Akteuren konstatiert, dass die Koordination von Innovationsprozessen, insbesondere für Akteure in kleineren Unternehmen und Organisationen, sehr voraussetzungsvoll ist und häufig wenig personelle und finanzielle Ressourcen zur Verfügung stehen. Insbesondere der Basischeck wurde zur Gewinnung eines Überblicks über Schwach- oder Leerstellen sowie zur Identifizierung von unmittelbarem Handlungsbedarf als sehr hilfreich angesehen. Aber auch die Möglichkeiten, einzelne Themen zu vertiefen, wurde von den Akteuren geschätzt, wenngleich teilweise Skepsis geäußert wurde, ob eine intensivere Auseinandersetzung mit den jeweils komplexen Themen im Alltag realistisch ist. Da häufig der Wunsch geäußert wurde, dass die Nutzung des ginkoo-Innovationsnavigators in entsprechende Beratungs- und Weiterbildungsangebote integriert werden sollte, werden zukünftige Möglichkeiten der Weiterentwicklung derzeit in Gesprächen mit verschiedenen Organisationen sondiert.

\section{Anmerkung}

[1] Das ginkoo-Projekt wurde von 2015 bis $2019 \mathrm{im}$ Rahmen des BMBFProgramms Innovationsgruppen für ein Nachhaltiges Landmanagement in Kooperation zwischen mehreren wissenschaftlichen Einrichtungen und Praxisakteuren durchgeführt. Mehr Informationen unter www.ginkoo. projekt.de. Der digitale ginkoo-Innovationsnavigator kann unter https:// www.ginkoo-innovationsnavigator.de/ genutzt werden. Er wurde außerdem als Broschüre veröffentlicht (König et al. 2019).

Der ginkoo-Innovationsnavigator wurde im ginkoo-Projekt erarbeitet. Neben den Autorinnen haben daran Rosemarie Siebert, Maria Busse, Katharina Diehl, Felix Zoll, Lukas Wortmann, Benjamin Nölting, Sven Lundie, Anita Beblek, Sven Möller, Nico Heitepriem, Michael Petschik, Ute Günster und Moritz Bor mitgewirkt. An der Validierung waren zudem Jan-Hendrik Skroblin, Jörg Große-Lochtmann, Susanne HofmannSouki beteiligt. Die Autorinnen haben zusätzlich Integrations- und Umsetzungsarbeiten geleistet.

[2] Vertiefende Informationen zu dem Navigator sind unter www.ginkooinnovationsnavigator.de abrufbar.

\section{AUTORINNEN + KONTAKT}

Dr. Martina Schäfer ist Professorin und wissenschaftliche Geschäftsführerin des Zentrums Technik und Gesellschaft der Technischen Universität Berlin.

Zentrum Technik und Gesellschaft der Technischen Universität Berlin (ZTG), Sekr. HBS 1, Hardenbergstr. 16-18, 10623 Berlin. Tel.: +49 30 31426854, E-Mail: schaefer@ztg.tu-berlin.de, Website: www.tu-berlin.de/ztg/menue/startseite_ztg/

Dr. Bettina König ist Mitglied am IRI THESys an der Humboldt-Universität zu Berlin, wo sie von 2014 bis 2019 das ginkoo-Projekt leitete. Sie ist an der Hochschule für nachhaltige Entwicklung in Eberswalde tätig.

IRI THESys - Integratives Forschungsinstitut zur Transformation von Mensch-Umwelt-Systemen, Humboldt-Universität zu Berlin, Friedrichstr. 181, 10117 Berlin. E-Mail: bettina.koenig@agrar.hu-berlin.de, Website: www.iri-thesys.org

Anett Kuntosch forschte an der HumboldtUniversität zu Berlin am IRI THESys zum Thema landwirtschaftliche Innovationsprozesse und -systeme sowie Wissensmanagement.

E-Mail: anett.kuntosch@agrar.hu-berlin.de

Dr. Beate Richter forschte an der Humboldt Universität zu Berlin am interdiziplinären Forschungsinstitut IRI THESys zu Nachhaltigkeitsthemen.

Tamara Schaal ist wissenschaftliche Mitarbeiterin an der Leuphana Universität Lüneburg.

Leuphana Universität Lüneburg, Institut für Ökologie, Universitätsallee 1, 21335 Lüneburg. Tel.: +49 4131 6774032, E-Mail: schaal@leuphana.de,

Webseite: www.leuphana.de/institute/institut-fueroekologie/personen/tamara-schaal.html
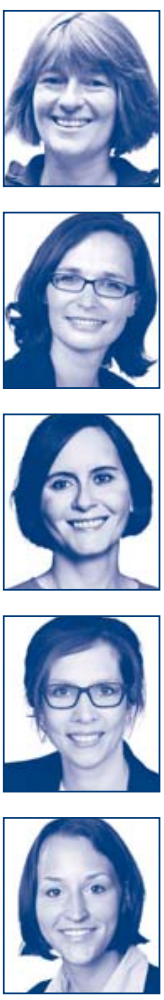


\section{Literatur}

Albers, S. (2005): Diffusion und Adoption von Innovationen. In: Albers S., Gassmann, O. (Hrsg.): Handbuch Technologie- und Innovationsmanagement. Wiesbaden, Gabler.

Belz, F. M./Schrader, U. (2012): Nachhaltigkeitsinnovationen durch Nutzerintegration? In: Beck, G./Kropp, C. (Hrsg.): Gesellschaft innovativ. Wiesbaden, Springer VS.

Blok, V./Gremmen, B./Wesselink, R. (2016): Dealing with the Wicked Problem of Sustainability in advance: The Role of Individual Virtuous Competence. In: Bus. Prof. Ethics J. 34/3: 297-327.

Busse, M./Siebert, R. (2017): The role of consumers in food innovation processes. In: European Journal of Innovation Management: 20-43.

Busse, M./Heitepriem, N./Siebert, R. (2019a): The Acceptability of Land Pools for the Sustainable Revalorisation of Wetland Meadows in the Spreewald Region, Germany. In: Sustainability 11: 4056

Busse, M./Siebert, R./Heitepriem, N. (2019b): Acceptability of innovative biomass heating plants in a German case study - a contribution to cultural landscape management and local energy supply. In: Energy, Sustainability and Society 9.

Busse, M./Fienitz, M./Fienitz, M./Siebert, R. (2019 c): Leitfaden zum Tool, Akzeptanz strategisch steigern' - ein Onlinetool fürs nachhaltige Landmanagement. In: ginkoo Projektberichte, Humboldt-Universität zu Berlin.

Chesbrough, H.W. (2006): Open Innovation: Researching a New Paradigm. New York, Oxford University Press.

Clausen, J./Fichter, K. (2019): The diffusion of environmental product and service innovations: Driving and inhibiting factors. In: Environmental Innovation and Societal Transitions 31: 64-95.

Corsten, H./Gössinger, R./Müller-Seitz, G./Schneider, H. (2016): Grundlagen des Technologie- und Innovationsmanagements. München, Vahlen.

Dhanaraj, C./Parkhe, A. (2006): Orchestrating innovation networks. In: Academy of Management Review 31/3: 659-669.

Fichter, K. (2006): Innovation Communities: Die Rolle von Promotionsnetzwerken bei Nachhaltigkeitsinnovtionen. In: Pfriem, R. et al. (Hrsg.): Innovationen für eine nachhaltige Entwicklung. Wiesbaden. Deutscher Universitätsverlag. 287-300.

Fichter, K. (2005): Modelle der Nutzerintegration in den Innovationsprozess Möglichkeiten und Grenzen der Integration von Verbrauchern in Innovationsprozesse für nachhaltige Produkte und Produktnutzungen in der Internetökonomie. IZT-WerkstattBericht.

Garud, R./Tuertscher, P./Van de Ven, A. H. (2013): Perspectives on Innovation Processes. The Academy of Management Annals 7/1: 773-817.

Geels, F. W./Schot, J. (2007): Typology of sociotechnical transition pathways. In: Res. Policy 36: 399-417.

Hirsch-Kreinsen, H. (2002): Unternehmensnetzwerke revisited. In: Zeitschrift für Soziologie 31/2: 106-124.

Hofbauer, G./Körner, R./Nikolaus, U./Poost, A. (2009): Marketing von Innovationen. Strategien und Mechanismen zur Durchsetzung von Innovationen. Stuttgart, Kohlhammer.

Jänicke, M. (2008): Megatrend Umweltinnovationen. Zur ökologischen Modernisierung von Wirtschaft und Staat. München, Oekom.

Ingram, J. (2015): Framing niche-regime linkage as adaptation: An analysis of learning and innovation networks for sustainable agriculture across Europe. In: Journal of Rural Studies 40: 59-75.

Hurmelinna-Laukkanen, P./Nätti, S. (2018): Orchestrator types, roles and capabilities. A framework for innovation networks. In: Industrial Marketing Management 74: 65-78.

Klewitz, J./Hansen, E. G. (2014): Sustainability-oriented innovation of SMEs: a systematic review. In: Journal of Cleaner Production 65: 57-75.

Knierim, A./Koutsouris, A./Mathé, S./Ndah, T. H./Temple, L./Triomphe, B./ Wielinga, E. (2015): Support to innovation processes: a theoretical point of departure. WP 1, deliverable 1.2 report of the AgriSPIN project.

König, B./Schäfer, M./Schaal, T./Kuntosch, A./Richter, B. (2019): Innovationsprozesse für nachhaltige Entwicklung integrativ managen - der ginkooInnovationsnavigator. In: ginkoo Projektberichte, Humboldt-Universität zu Berlin.

Koller, H./Langmann, C./Untiedt, H. M. (2006): Das Management von Innovationsnetzwerken in verschiedenen Phasen. In: Wodjaand, F./Barth, A.
(Hrsg.): Innovative Kooperationsnetzwerke. Wiesbaden, Deutscher Universitäts-Verlag. 29-80.

Kuntosch, A./König, B./Bokelmann, W./Doernberg, A./Siebert, R./Schwerdtner, W./Busse, M. (2020): A system study to identify adoption barriers for implementation of eco- efficient innovation in small and medium-sized horticultural enterprises. In: Horticulturae 33/6: 1-21.

Kuntosch, A. (2019): Roadmap Wissensmanagement. Wissen als Ressource für Innovationsprozesse im nachhaltigen Landmanagement nutzbar machen. In: ginkoo Projektberichte, Humboldt-Universität zu Berlin.

Lettkemann, E. (2018): Das Feuerwerksmodell der Innovation. In: Handbuch Innovationsforschung. Wiesbaden, Springer. 1-13.

Levén, P./Holmström, J./Mathiassen, L. (2014): Managing research and innovation networks: Evidence from a government sponsored cross-industry program. In: Research Policy 43/1: 156-168.

Leydesdorff, L./Etzkowitz, H. (1996): Emergence of a Triple Helix of University-Industry-Government Relations. In: Science and Public Policy 23, 279-286.

Mclver, D./Lengnick-Hall, C. (2018): The causal ambiguity paradox: Deliberate actions under causal ambiguity. In: Strategic Organization 16/3: 304-322.

Möller, K./Kubach, M./Bizer, K./Krüger, L. (2011): Nachhaltigkeitsorientierte Bewertung von Innovationsprojekten. Göttingen, Cuvillier.

Ndah, T. H./Knierim, A./Faure, G./Zarokosta, E./Audouin, S./Wielinga, E./ Koutsouris, A./Heanue, K./Temple, L./Triomphe, B./Kelly, T. (2017): A Scientific Report on Cross Compared Research Insights on Innovation Support Practices. AgriSpin Deliverable 3.1. Stuttgart, Uni-Hohenheim.

Nölting, B./Schäfer, M. (2020): Framing cooperation as a key element for sustainable agriculture and food production: an analytical framework. ZTG-Discussion Paper Nr. 43/2020

Orenstein, D. E./Shach-Pinsley, D. (2017): A Comparative Framework for Assessing Sustainability Initiatives at the Regional Scale. In: World Dev. 98: 245-256.

Peterson, H.-C./Mager, S. E. (2011): From motivating assumptions to a practical innovation model. In: Latesteijn, H.-C./Andeweg, K. (Hrsg.): The Transforum Model: Transforming Agro Innovation toward Sustainable Development. Wiesbaden, Springer Verlag. 97-127.

Probst, G./Romhardt, K./Raub, S. (2012): Wissen managen - Wie Unternehmen ihre wertvollste resource optimal nutzen. Wiesbaden, Springer.

Pronay, S./Buzas, N. (2015): The Evolution of Marketing Influence in the Innovation Process: Toward a New Science-to-Business Marketing Model in Quadruple Helix. In: Journal of the Knowledge Economy 6: 494-504.

Rammer, C./Gottschalk, S./Peters, B./Bersch, J./Erdsiek, D. (2016): Die Rolle von KMU für Forschung und Innovation in Deutschland. Studie im Auftrag der Expertenkommission Forschung und Innovation. Studien zum deutschen Innovationssystem Nr. 10/2016. Mannheim, Zentrum für Europäische Wirtschaftsforschung.

Reed, M. S./Fraser, E. D. G./Dougill, A. J. (2006): An adaptive learning process for developing and applying sustainability indicators with local communities. In: Ecol. Econ. 59: 406-418.

Richter, R./Fink, M./Lang, R./Maresch, D. (2020): Social Entrepreneurship and Innovation in Rural Europe. London, Routledge.

Richter, R. (2019): Rural social enterprises as embedded intermediaries: The innovative power of connecting rural communities with supra-regional networks. In: Journal of Rural Studies 70: 179-187.

Richter, B./König, B. (2019): Marketing-Manual für das nachhaltige Landmanagement. Integration und Umsetzung von Marketing in Innovationsprozessen. In: ginkoo Projektberichte, Humboldt-Universität zu Berlin.

Schäfer, M./König, B. (2018): The role of cooperation for sustainability innovations in the agriculture and food sector. In: International Journal of Agricultural Extension 6/3: 65-78.

Schäfer, M./Nölting, B./Schaal, T./Zscheischler, J. (2019): Leitfaden Kooperationsmanagement für Nachhaltigkeitsinnovationen. ginkoo Projektberichte, Humboldt-Universität zu Berlin.

Schroeder, R. G./Van der Ven, A. H./Scudder, G. D./ Polley, D. (2000): The development of innovation ideas. In: Van der Ven, A. H. et al. (2000): Research on Management of Innovation. The MIRP Studies. New York, Oxford University Press. 
Steinhoff, F./Trommsdorf (2011): Innovation Marketing: An Introduction. In: Hülsmann, M., Pfeffermann, N.: Strategies and Communications for Innovations. An Integrative Management View for Companies and Networks. Berlin, Springer. 105-116.

Sydow J. (2006): Über Netzwerke, Allianzsysteme, Verbünde, Kooperationen und Konstellationen. Wiesbaden, Gabler.

Sydow, J. (1992): Strategische Netzwerke. Evolution und Organisation. Wiesbaden, Gabler.

Sydow, J./Braun, T. (2018): Projects as temporary organizations: An agenda for further theorizing the interorganizational dimension. In: International Journal of Project Management 36/1: 4-11.

Trommsdorff, V./Steinhoff, F. (2013): Innovationsmarketing. München, Verlag Franz Vahlen.

Trommsdorff, V./Binsack M. (1999) Informationsgrundlagen für das Innovationsmarketing. In: Tintelnot C./Meißner D./Steinmeier I. (Hrsg.): Innovationsmanagement. Innovations- und Technologiemanagement. Berlin, Springer.

Van de Ven, A. (1986): Central problems in the management of innovation. In: Management Science 32/5: 590-607.
Van de Ven, A. H./Polley, D./Garud, R./Venkataraman, S. (1999): The innovation journey. New York: Oxford University Press.

Van de Ven, A./Engleman, R. (2004): Central problems in managing corporate innovation and entrepreneurship. In: Advances in Entrepreneurship, Firm Emergence and Growth 7: 47-72.

von Ahsen A./Heesen M./Kuchenbuch A. (2010): Grundlagen der Bewertung von Innovationen im Mittelstand. In: Ahsen, A. (Hrsg.) Bewertung von Innovationen im Mittelstand. Berlin, Springer.

Wielinga, H. E./Dijkshoorn, M./Sol, J. (2010): Structural Innovations in the Dutch Green Knowledge System. In: Darnhofer, I. (Hrsg.): Proceedings of the 9th European IFSA Symposium. Wien.

Weiber, R./Pohl, A. (2017): Marketing und Innovation. Stuttgart, Kohlhammer. Zoll, F./Diehl, K./Siebert, R. (2020): Leitfaden zum Tool Identification of Common Sustainability Goals (ICO) - Ein Tool zur Identifizierung gemeinsamer Nachhaltigkeitsziele. In: ginkoo Projektberichte, HumboldtUniversität zu Berlin.

Zoll, F./Diehl, K./Siebert, R. (2019): Integrating Sustainability Goals in Innovation Processes: Applying a Decision Support Tool in a Dual-Purpose Chicken Case Study. In: Sustainability 2019 11/14: 3761. 